\title{
Two De-Noising Methods Based on Gabor Transform
}

\author{
Yongjun Shen ${ }^{1, a}$, Guangming Zhang ${ }^{1, b}$, Shaopu Yang ${ }^{1, c}$ and Haijun Xing ${ }^{1, d}$ \\ ${ }^{1}$ Department of Mechanical Engineering, Shijiazhuang Tiedao University, No. 17 Bei Erhuan Dong \\ Road, Shijiazhuang 050043, China
}

ashenyongjun@126.com, ${ }^{b}$ ming5819205@sina.com, cyangsp@stdu.edu.cn, dxinghj@stdu.edu.cn

\section{Keywords: Gabor Transform, De-noising Method, Wavelet Transform, Fault Diagnosis}

\begin{abstract}
Two de-noising methods, named as the averaging method in Gabor transform domain (AMGTD) and the adaptive filtering method in Gabor transform domain (AFMGTD), are presented in this paper. These two methods are established based on the correlativity of the source signals and the background noise in time domain and Gabor transform domain, that is to say, the uncorrelated source signals and background noise in time domain would still be uncorrelated in Gabor transform domain. The construction and computation scheme of these two methods are investigated. The de-noising performances are illustrated by some simulation signals, and the wavelet transform is used to compare with these two new de-noising methods. The results show that these two methods have better de-noising performance than the wavelet transform, and could reduce the background noise in the vibration signal more effectively.
\end{abstract}

\section{Introduction}

Generally the measured vibration signals contain important information for the prognostic and fault diagnosis purposes. However, the unavoidable background noise may influence, or even distort the diagnosis results. Thus the research on de-noising methods for vibration signals has attracted much attention and many valuable works were presented over the past years. The Fast Fourier Transform (FFT) and other FFT-based spectrum analysis methods, of course, are the most commonly used signal analysis methods for fault diagnosis, which could tell us some useful information about the frequency contents and their magnitude relations, so as to help us understand the system condition [1-2]. Unfortunately, the FFT and other FFT-based methods are only suitable for stationary signals whose frequency contents do not change over time, and could not present satisfactory results if the vibration signals are non-stationary. For example, when the running speed of a machine is varied, such as speedup or speed-down, its bandwidth in frequency domain would become much wider which may present less helpful information.

Accordingly, a lot of time-frequency methods suitable for non-stationary signal, such as wavelet transform, Wigner distribution, Gabor transform, etc., have been applied in fault diagnosis [3-11]. For example, Peng and Chu [3] presented a summary about the application of the wavelet transform in machine fault diagnosis, and gave some useful prospects of the wavelet transform combined with other tools in condition monitoring and fault diagnosis. Wang and Mcfadden [5] applied the wavelet transform to represent all possible types of transients in vibration signals generated by faults in a helicopter gearbox. Gelle and Colas [9] gave preliminary research on the application of Blind Source Separation (BSS) in fault diagnosis. Roan, etc. [10] presented a non-linear BSS approach and applied it to failure detection of gear tooth. Shen and Yang [11] presented a novel BSS method based on Fractional Fourier Transform, which could be used to separate the mixed non-stationary signals successfully and was applied for fault diagnosis of rolling bearing in freight train successfully.

In these de-noising methods based on time-frequency, an unavoidable problem is to select the appropriate threshold values in time-frequency plane. As we all know, the undesirable threshold values would deteriorate the de-noising effect. In this paper, two de-noising methods without consideration on the selection of threshold values are presented, which are based on the Gabor transform. The first method is the averaging method in Gabor transform domain (AMGTD), where the de-noised signal could be obtained by applying inverse Gabor transform to the averaged signal in Gabor transform domain through many groups of measured signals. The second one is named as the 
adaptive filtering method in Gabor transform domain (AFMGTD), which means that one could adaptively obtain the threshold values in Gabor transform domain by averaging many groups of measured signals.

\section{Gabor Transform Principle}

The Gabor transform was proposed in 1946 by Gabor to perform simultaneous time-frequency analysis of signals. After that many researchers improved the computation method of Gabor transform and its inverse transform, and some de-noising methods based on Gabor transform were presented [12-13]. Based on the Gabor transform, the union time-frequency function of a non-stationary signal could be established by the time and frequency shift. Moreover, the union time-frequency function could be expressed by other two separated sampling mesh parameters (m and $n$ ) in time-frequency plane, where these two sampling mesh parameters are related with time $t$ and frequency f respectively.

The continuous Gabor expansion of the signal $s(t)$ is defined as

$$
s(t)=\sum_{m=-\infty}^{\infty} \sum_{n=-\infty}^{\infty} a_{m n} g_{m n}(t),
$$

where $a_{m n}$ is the continuous Gabor coefficients and $g_{m n}(t)$ is the basis function denoted by

$$
g_{m n}(t)=g(t-m T) e^{j n \Omega t} .
$$

Here the time shift $T$ and the frequency shift $\Omega$ satisfy the relationship $\Omega=2 \pi / T$, and $m$ and $n$ may take all integer values. The continuous Gabor coefficients is

$$
a_{m n}=\int_{-\infty}^{\infty} s(t) \gamma_{m n}^{*}(t) d t
$$

where $\gamma_{m n}^{*}(t)$ is complex conjugation of $\gamma_{m n}(t)$, and $\gamma_{m n}(t)$ is dual function of Gabor basis function $g_{m n}(t)$. In Gabor transform, the following bi-orthonormality condition must be satisfied

$$
\int g_{m n}(t) \gamma_{m n}^{*}(t-m T) e^{-j n \Omega t} d t=\delta(m) \delta(n) .
$$

For a discrete periodic signal $s(k)$, namely $s(k)=s(k+L)$, the discrete Gabor expansion is given in the following way ${ }^{[12-13]}$

$$
s(k)=\sum_{m=0}^{M-1} \sum_{n=0}^{N-1} a_{m n} g(k-m T) e^{j n \Omega k},
$$

and the discrete Gabor coefficients is

$$
a_{m n}=\sum_{k=0}^{L-1} s(k) \gamma^{*}(k-m T) e^{-j n \Omega k},
$$

where $T$ and $\Omega$ is the time and frequency shift, $M$ and $N$ is the frequency and the time sampling total number. Obviously, Gabor transform is a kind of linear transform. More detailed materials about the computation schemes of discrete Gabor transform could be found in the famous references [12-13].

\section{De-noising Methods based on Gabor Transform}

Averaging Method in Gabor Transform Domain (AMGTD)Numbers. We could show the configuration of AMGTD by a group of signals $z_{j}(t)$ with equal length, which are composed of the source signal $y(t)$ and noise $N_{j}(t)$, 


$$
z_{j}(t)=y(t)+N_{j}(t), j=1,2, \cdots, l,
$$

where $y(t)$ and all $N_{j}(t)$ are mutually uncorrelated. Because Gabor transform is linear, they would still be mutually uncorrelated in Gabor transform domain.

The first step is to compute the Gabor transform of $z_{j}(t)$, and one could obtain a group of time-frequency signals $Z_{j}(m, n)$ in Gabor domain

$$
Z_{j}(m, n)=\operatorname{dgt}\left[z_{j}(t)\right], j=1,2, \cdots, l,
$$

where $\operatorname{dgt}[]$ is the Gabor transform operator.

Now we could observe the characteristics of some typical signals in the Gabor time-frequency domain. Without generality, supposing that a signal $s(t)$ is combination of two harmonic signals, that means $s(t)=e^{j w_{1} t}+e^{j w_{2} t}$, and selecting the dual function $\gamma_{m n}(t)=\left(\frac{a}{T}\right)^{2} e^{j w t}$, one could obtain the Gabor coefficients

$$
a_{m n}=\int_{-\infty}^{\infty} s(t) \gamma_{m n}^{*}(t) d t=2 \pi(a / T)^{2}\left[\delta\left(w-w_{1}\right)+\delta\left(w-w_{2}\right)\right]
$$

Obviously, the Gabor coefficients of signal $s(t)$ are two belt-shaped impulse functions along the two frequency lines of signal $s(t)$. The result shows that the periodic signal generally distributes in some narrow bands in Gabor time-frequency plane, so that the signal energy would be very concentrated in the bands. And the similar research shows that other narrow-band signals, such as modulated signals, have the same property. However, the white noise signal shows disorderly distribution in Gabor time-frequency plane, which means the energy of the noise would bestrew the whole Gabor time-frequency plane.

According to the above situations, one could establish the second step of AMGTD. That means, the average for the groups of Gabor coefficients $Z_{j}(m, n)$ could be computed by

$$
\bar{Z}(m, n)=\frac{\sum_{j=1}^{l} Z_{j}(m, n)}{l} .
$$

Because the source signal $y(t)$ and $N_{j}(t)$ are mutually uncorrelated in time domain and in Gabor transform domain, $\operatorname{dgt}[y(t)]$ would be the principal component of $\bar{Z}(m, n)$ while $\operatorname{dgt}\left[N_{j}(t)\right]$ would be very small when $l$ becomes very large.

Then the coefficients $\bar{Z}(m, n)$ could be transformed into time domain by inverse Gabor transform, and one could obtain the de-noised source signal $\bar{y}(t)$. This is the third step of AMGTD. Adaptive Filtering Method in Gabor Transform Domain (AFMGTD). Similar to the above section, the configuration of the AFMGTD is introduced through the same group of signals $z_{j}(t)$. Applying Gabor transform to $z_{j}(t)$, one could obtain $Z_{j}(m, n)$, the real part $Z_{j}^{R}(m, n)$ and the imaginary part $Z_{j}^{I}(m, n)$ of $Z_{j}(m, n)$ respectively

$$
Z_{j}(m, n)=\operatorname{dgt}\left[z_{j}(t)\right], Z_{j}^{R}(m, n)=\operatorname{real}\left[Z_{j}(m, n)\right], Z_{j}^{I}(m, n)=\operatorname{imag}\left[Z_{j}(m, n)\right] .
$$

Then the second step is to construct the adaptive filter shown as

$$
H_{R}(m, n)=\frac{\sum_{j=1}^{l} \sum_{k \neq j}^{l}\left(Z_{j}^{R}(m, n) \cdot Z_{k}^{R}(m, n)\right)}{(l-1) \sum_{j=1}^{l}\left(Z_{j}^{R}(m, n) \cdot Z_{j}^{R}(m, n)\right)}, H_{I}(m, n)=\frac{\sum_{j=1}^{l} \sum_{k \neq j}^{l}\left(Z_{j}^{I}(m, n) \cdot Z_{k}^{I}(m, n)\right)}{(l-1) \sum_{j=1}^{l}\left(Z_{j}^{I}(m, n) \cdot Z_{j}^{I}(m, n)\right)},
$$

Expanding the numerator in Eq. (11) one could obtain

$$
\sum_{j=1}^{l} \sum_{k \neq j}^{l}\left(Z_{j}^{R} \cdot Z_{k}^{R}\right)=\sum_{j=1}^{l} \sum_{k \neq j}^{l}\left\{Y^{R} \cdot Y^{R}+Y^{R} \cdot W_{j}^{R}+W_{j}^{R} \cdot Y^{R}+W_{j}^{R} \cdot W_{j}^{R}\right\},
$$




$$
\sum_{j=1}^{l} \sum_{k \neq j}^{l}\left(Z_{j}^{I} \cdot Z_{k}^{I}\right)=\sum_{j=1}^{l} \sum_{k \neq j}^{l}\left\{Y^{I} \cdot Y^{I}+Y^{I} \cdot W_{j}^{I}+W_{j}^{I} \cdot Y^{I}+W_{j}^{I} \cdot W_{j}^{I}\right\},
$$

where $Y^{R}=\operatorname{real}[\operatorname{dgt}[y(t)]], W_{j}^{R}=\operatorname{real}\left[\operatorname{dgt}\left[N_{j}(t)\right]\right], Y^{I}=\operatorname{imag}[\operatorname{dgt}[y(t)]], W_{j}^{I}=\operatorname{imag}\left[\operatorname{dgt}\left[N_{j}(t)\right]\right]$.

According to the aforementioned property of Gabor transform, it could be concluded that the last three parts in Eq. (12a) and Eq. (12b) would be very small when $l$ becomes very large, and only the first part determined by the source signal $y(t)$ would be kept. Then one obtain a filter denoted by $H_{R}(m, n)$ and $H_{I}(m, n)$, which matches the source signal $y(t)$ very well. Here the term "adaptive" means that the filter is obtained only based on the measured signals.

At the end one could randomly select one element from $Z_{j}(m, n)$, such as $Z_{1}(m, n)$, multiply its real part and imaginary part by the new filter $H_{R}(m, n)$ and $H_{I}(m, n)$ respectively, and transform the result into time domain by inverse Gabor transform. Then one would obtain the de-noised signal $\bar{y}(t)$.

Discussion on These Two Methods. From the description of these two new methods, one could find there is a key problem to overcome. The problem is how to obtain the group of signals $z_{j}(t)$. Here we give two possible ways. One way is to measure a longtime signal and then divide it into a group of signals with equal length based on the known period, if the source signal $y(t)$ is stationary or cycle-stationary. The other way is to directly measure a group of signals if the source signal $y(t)$ is non-stationary. Of course, in this measurement process one must keep the measurement condition unchanged and adopt the same sampling parameters.

\section{Illustration of the Two Methods}

In order to illustrate the performance of these two new methods, we research two examples and compare the performance of the methods with that of the wavelet transform, where we adopt the following function in wavelet transform toolbox of MatLab 7.0

$$
[\mathrm{xd}, \mathrm{cxd}, 1 \mathrm{xd}]=\operatorname{wden}\left(z_{j}(t),\right. \text { 'rigrsure', 's', 'sln', 5,'db10'). }
$$

De-noising of Simulation Signal. The selected simulation signal is shown as

$$
z(t)=5 \sum_{n=1}^{4} \sin (2 \pi 100 n t)+4 \sin (2 \pi 500 t)+k \cdot N(t)
$$

where the sum of five periodic signals is considered as the aforementioned source signal $y(t), N(t)$ is the Gaussian noise with zero mean and unit variance, and the constant $k$ denotes the level of noise energy. The sampling time is $10 \mathrm{~s}$ and the sampling frequency is $10 \mathrm{KHz}$. Then one could equably divide $z(t)$ into 100 segments so as to obtain $z_{j}(t)$.

The SNRs of the source signal with noise, the de-noised signal by wavelet transform, the de-noised signal by AMGTD and the de-noised signal by AFMGTD are shown in Fig. 1 by “ $\bigcirc$ ", “ $\square$ ", " $\diamond$ " and " $\Delta$ " under different noise level $k$ respectively. The time history of source signal $y(t)$ and its frequency spectrum are shown in Fig. 2. In more detail, the time histories and frequency spectra of the source signal with noise, the de-noised one by wavelet transform, the de-noised one by AMGTD and the de-noised one by AFMGTD are shown in Fig. 3 under the noise level $k=20$.

From these figures it could be concluded that the new methods have excellent de-noising performance than the traditional wavelet transform under different noise level. When the noise level is quite weak, one may obtain smooth de-noised signals in time domain using these three de-noising methods, and they are nearly the same as the source signal. With the increase of noise level, the time history of the de-noised signal by wavelet transform is much different from the source one, while the time histories of the de-noised signals by AMGTD and AFMGTD are similar to the source one. Moreover, these three de-noising methods have acceptable performance in frequency domain, and 
frequency lines obtained by AMGTD and AFMGTD are more clear-cut than that by wavelet transform. If the noise level becomes very large, the de-noising performance by wavelet transform is too bad to observe useful information either in time or frequency domain, while the frequency lines, even the time histories of the de-noised signals by AMGTD and AFMGTD could still tell us more information about the source signal.

\section{Conclusion}

According to the basic theory of Gabor transform, two de-noising methods named as AMGTD and AFMGTD based on Gabor transform are put forward. The de-noising performance of the two new methods are validated by simulation signal, and the results show that the new methods have better de-noising effect than wavelet transform. The two new methods could be applied in fault diagnosis, and may provide a new direction for fault diagnosis.

\section{Acknowledgement}

The authors are grateful to the support by National Natural Science Foundation of China (No. 11072158, 10602038 and 50625518), Natural Science Funds for Distinguished Young Scholar of Hebei Province (No. E2010002047), Key Project of Chinese Ministry of Education (No. 209013), the Program for Changjiang Scholars and Innovative Research Team in University (No. IRT0971), and "the Excellent Going Abroad Experts" Training Program in Hebei Province.

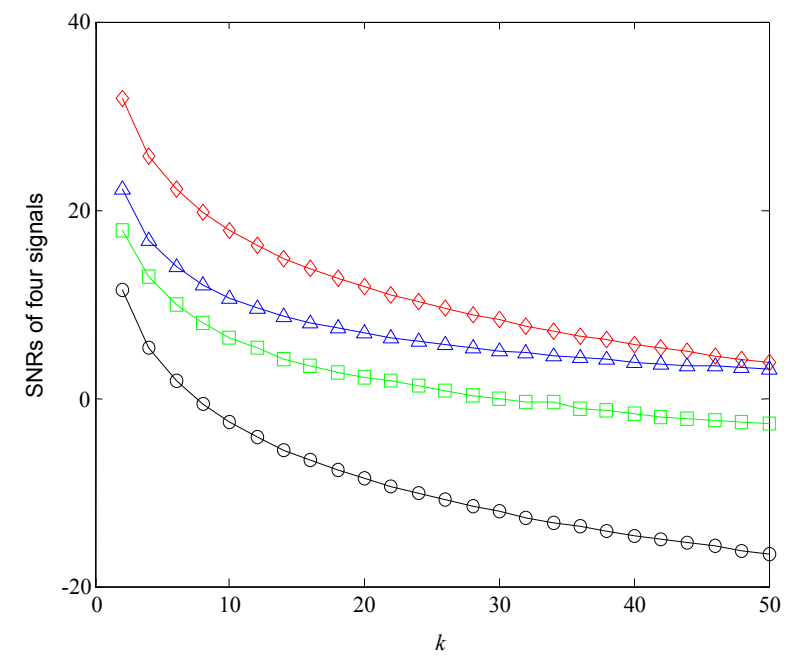

Fig. 1. The SNRs of four signals
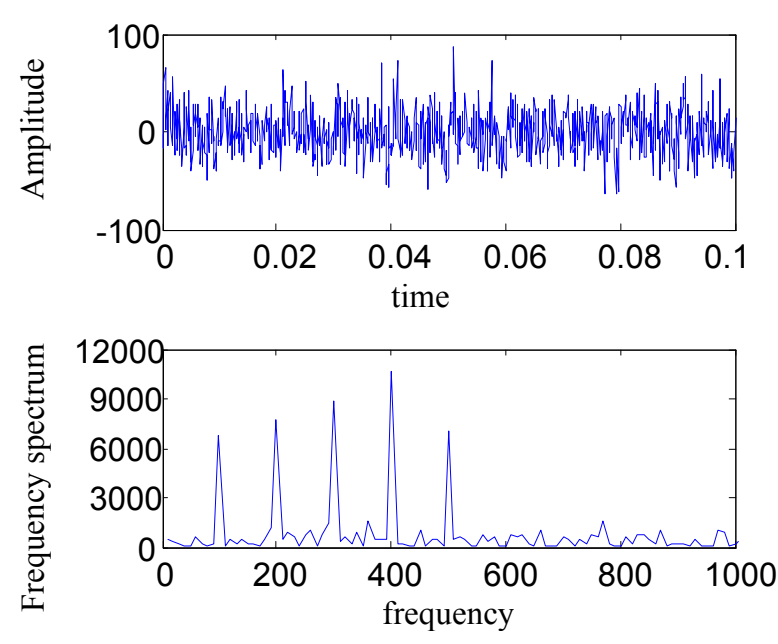

(a)
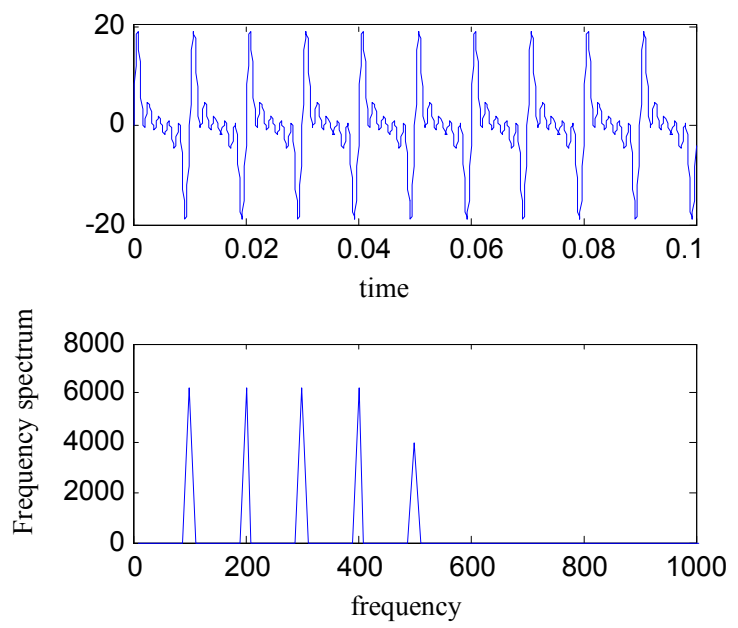

Fig. 2. The source signal and its frequency spectrum
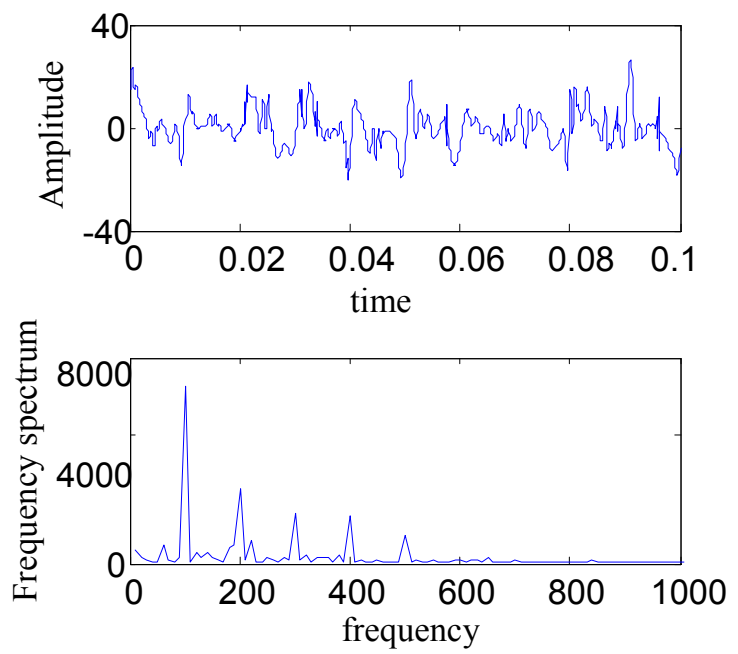

(b) 

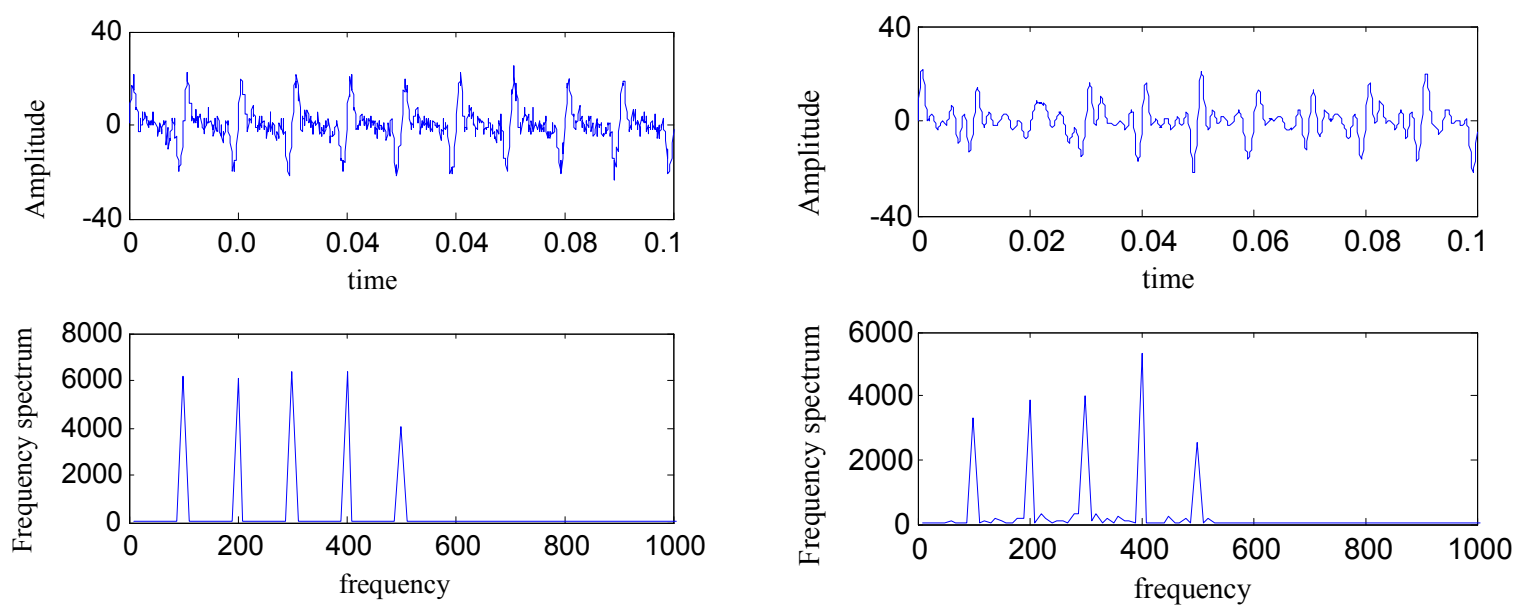

(c)

(d)

Fig. 3. The time histories and frequency spectra of four signals when $k=20:$ (a) the source signal with noise; (b) the de-noised signal by wavelet transform; (c) the de-noised signal by AMGTD; (d) the de-noised signal by AFMGTD

\section{Reference}

[1] R. B. Randall, A new method of modeling gear faults, ASME Journal of Mechanical Design, 104 (1982) 259-267.

[2] S. K. Lee, P. R. White, Higher-order time-frequency analysis and its application to fault detection in rotating machinery, Mechanical Systems and Signal Processing, 11(1997) 637-650.

[3] Z. Peng, F. Chu, Application of the wavelet transform in machine condition monitoring and fault diagnostics: a review with bibliography, Mechanical System and Signal Processing, 18(2004) 199-221.

[4] I. S. Bozchalooi, M. Liang, A smoothness index-guided approach to wavelet parameter selection in signal de-noising and fault detection, Journal of Sound and Vibration, 308(2007) 246-267.

[5] W. J. Wang, P. D. McFadden, Application of wavelets to gearbox vibration signals for fault detection, Journal of Sound and Vibration 192(1996) 927-939.

[6] C. K. Sung, H. M. Tai, C. W. Chen, Locating defects of a gear system by the technique of wavelet transform, Mechanism and Machine Theory, 35(2000) 1169-1182.

[7] J. Lin, L. Qu, Feature extraction based on morlet wavelet and its application for mechanical fault diagnosis, Journal of Sound and Vibration, 234(2000) 135-148.

[8] P. W. Tsea, W. X. Yang, H.Y. Tam, Machine fault diagnosis through an effective exact wavelet analysis, Journal of Sound and Vibration 277(2004) 1005-1024.

[9] G. Gelle, M. Colas, Blind Sources separation: a tool for rotating machine monitoring by vibrations analysis? Journal of Sound and Vibration, 248(2001) 865-885.

[10] M. J. Roan, J. G. Erling, L. H. Sibul, A new, non-linear, adaptive, blind source separation approach to gear tooth failure detection and analysis, Mechanical Systems and Signal Processing, 16(2002) 719-740.

[11] Y. Shen, S. Yang. A new blind-source-separation method and its application to fault diagnosis of rolling bearing, International Journal of Nonlinear Science and Numerical Simulation, 7(2006) 245-250.

[12] S. Qian, D. Chen, Discrete Gabor transform, IEEE Trans on Signal Processing, 41(1993) 2429-2438.

[13] J. Wexler, S. Raz, Discrete Gabor expansions, Signal Processing, 21(1991) 207-220. 